\title{
Computer technology to imitate traditional tie-dye patterns
}

\author{
Xiao-Sha Yin ${ }^{1, a}$ and Jian-Ming Wang ${ }^{2, b, \dagger}$ \\ ${ }^{1}$ Beijing Institute of Fashion Technology, Beijing, China \\ ${ }^{2}$ Beijing Institute of Fashion Technology, Beijing, China \\ am13051156739@163.com,bwangjm1502@163.com \\ $\dagger$ Jian-Ming Wang
}

\begin{abstract}
Because of its long tradition of printing and dyeing technology cycle, complicated production process, and the pollution of the environment, energy consumption in the future development of the ecological environment has hazards. By using Ultra Fractal to simulate fractal tie-dye pattern, reproduce the Chinese traditional tie-dye art, but also carry forward the traditional culture and inheritance.
\end{abstract}

Keywords: Simulation; Fractal; Tie-dye.

\section{Introduction}

Tie-dye is an ancient folk art, handmade tie-dye all over the world, different historical and geographical environment, create tie-dye works differently[1].Since the penetration of fiber texture of the fabric, the fabric will be a strong level of color halos, even if the same graphic design, because of different method, different tightness, different water temperatures will allow the works to produce dry-changing of the results, so there will be some limitations in the design, and computer simulation can be dyed just right to fill the vacancy[2].

In recent years, through the use of computer-aided design technology graphic design, fractal pattern design and intelligent graphic design and digital printing technology and other methods combined. Wherein the graphic design software, image processing software Photoshop, graphics software Freehand, Illustrator, Corel Draw, mapping software Painter, typesetting software Pagemkae, Quark, etc. [3]; commonly used software is mainly Apophysis and Ultra Fractal [4].

Fractal geometry, 1975 by B.B. Mandelbrot of IBM company was founded, with the principle of self-similarity, and insights on the phenomenon of chaos, the fine structure of its object of study as a complex disorder of nature and social activities in a wide range, and has a certain law system [5]. Fractal patterns in 
fashion design, decorative design, surface texture, pattern design, as well as a large number of Arts and crafts in pattern design can increase the artistic expression and influence of design objects, produce unexpected decorative effect. Fractal image exquisite and artistic styles, the screen is filled with overlapping, cascading, Warbler complex, winding, full of a wealth of surprises and gorgeous color [6].

This paper use Ultra Fractal designed to simulate the pattern of traditional tie-dyed.

\section{Ultra Fractal Simulation of Tie-Dye Patterns}

\section{Kaleidoscope formula}

The Kaleidoscope transformation fills the screen with rotated copies of a small radial slice of the fractal, creating a kaleidoscope effect. By tweaking the parameters, you can simulate many different kinds of symmetry. Try experimenting with the Center and Rotation angle parameters to obtain good results.

\section{The pixel fractal formula}

The Pixel fractal formula is a simple stub formula that marks all pixels in the layer as outside. This gives exclusive control over the layer to the coloring algorithm selected on the Outside tab of the Layer Properties tool window. Combined with a direct coloring algorithm such as the standard Image coloring algorithm, this makes it possible to import images.

\section{Applications clouds formula}

Many graphics software can automatically generate a pattern of clouds, cloud pattern color shades, soft transitions. Cloud pattern of the feature and in tie dye penetration process due to different color halo effect caused by similar, so cloud formula can be applied to simulate color in a tie-dyed pattern is not uniform and the halo effect. 


\section{Combined Kaleidoscope Formula, Pixel Formula and Clouds Formula}

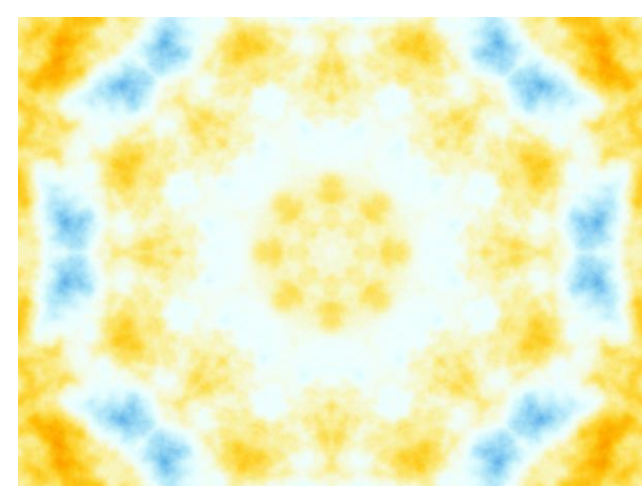

(a)

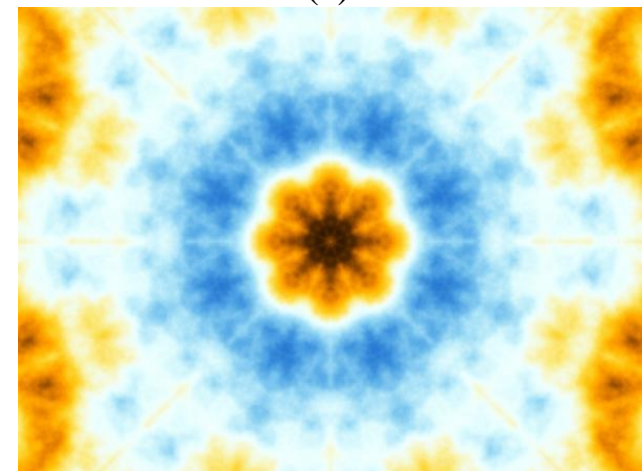

(b)

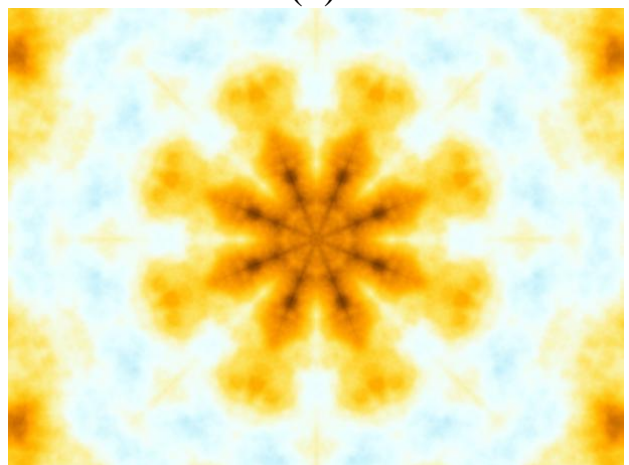

(c) 


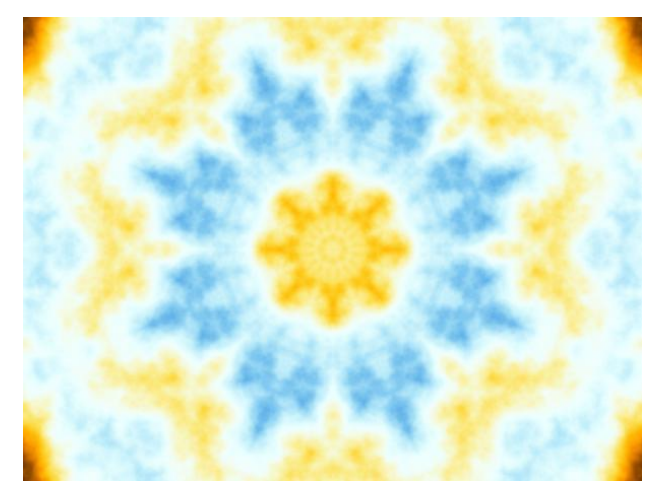

(d)

Fig.1. Ultra Fractal simulation imitation tie-dye pattern

Fig.1.(a) is in the Ultra Fractal "Mapping" the select Kaleidoscope formula, "Formula" select the pixel, "Outside" choose cloud formula of Fractional Brownian Motion; Fig. 1.(b) is "Outside" select Perlin Noise in the clouds formula; Fig. 1.(c) is "Outside" choose cloud formula of Perlin Noise D; Fig. 1.(d) is to select cloud 4D of plasma in the formula.

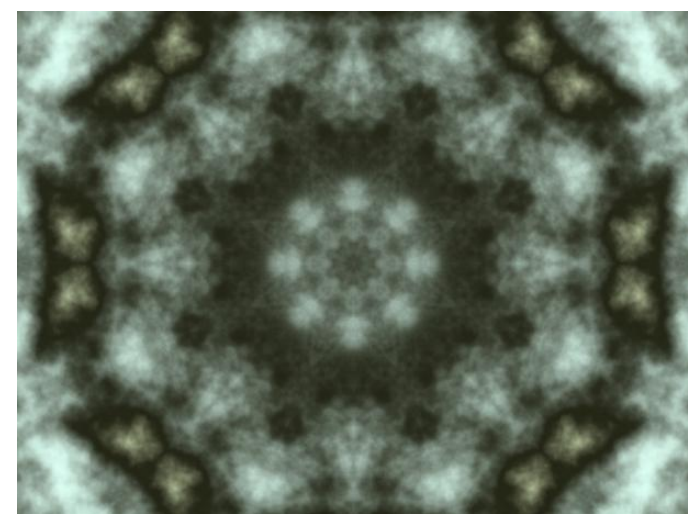

(a) 


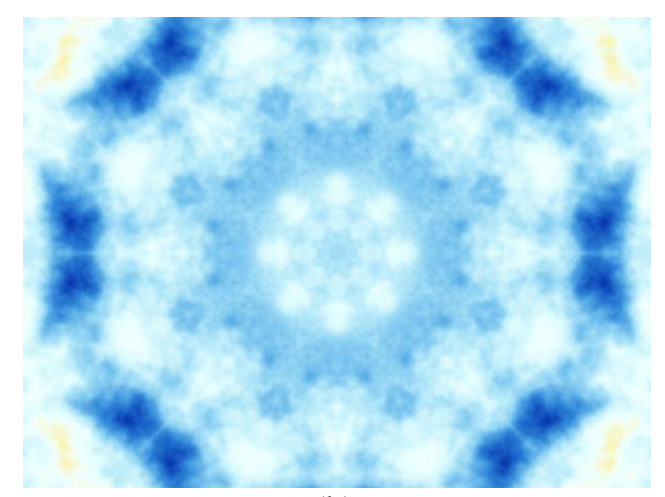

(b)

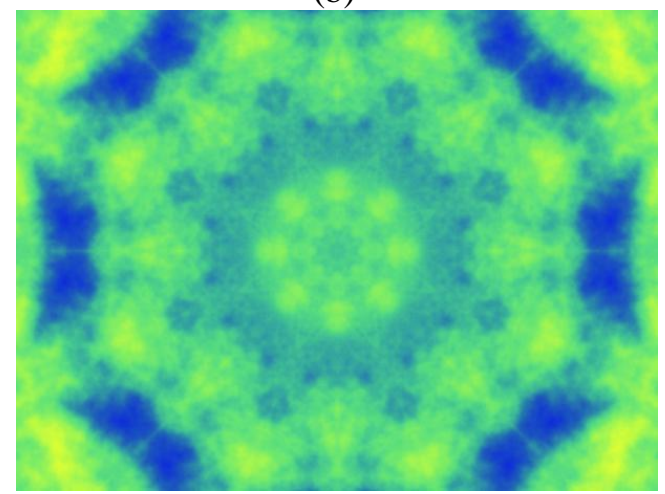

(c)

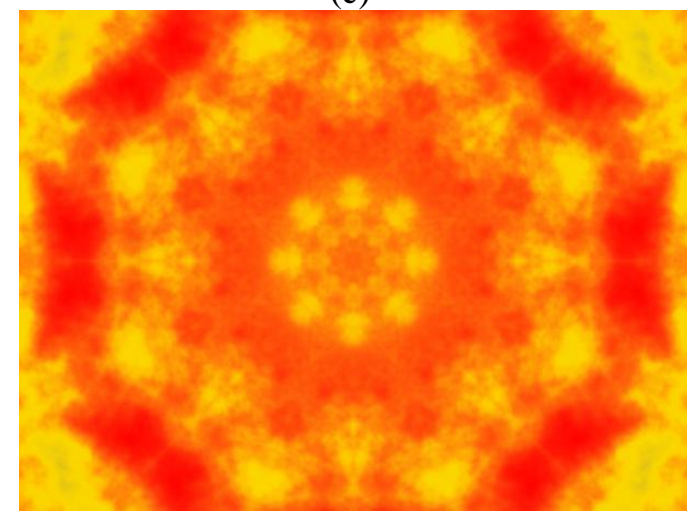

(d)

Fig 2 After adjusting the color of the tie dye patterns

Fig. 2.(a) and Fig. 2.(b) imitation monochrome tie-dye,adjusted Gradient Editor Color Control Point parameters to achieve monochrome tie-dye;Fig. 2 (c) and Fig. 2 (d) imitation color tie-dye by controlling the gradient editor to adjust the color transition points, tie-dye to achieve the desired state. 


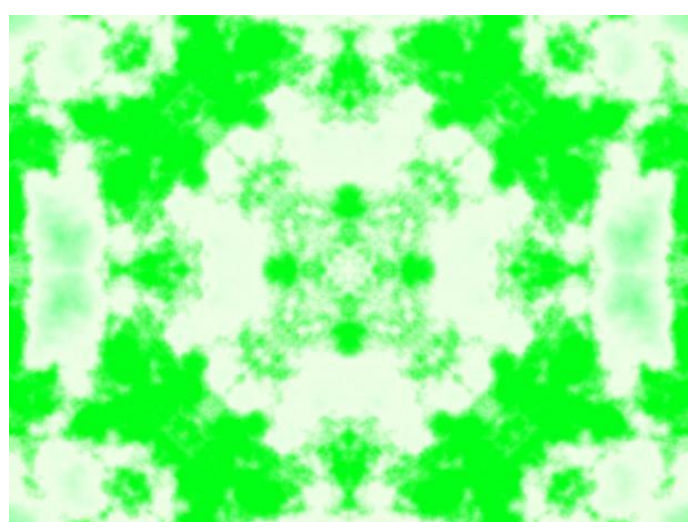

(a)

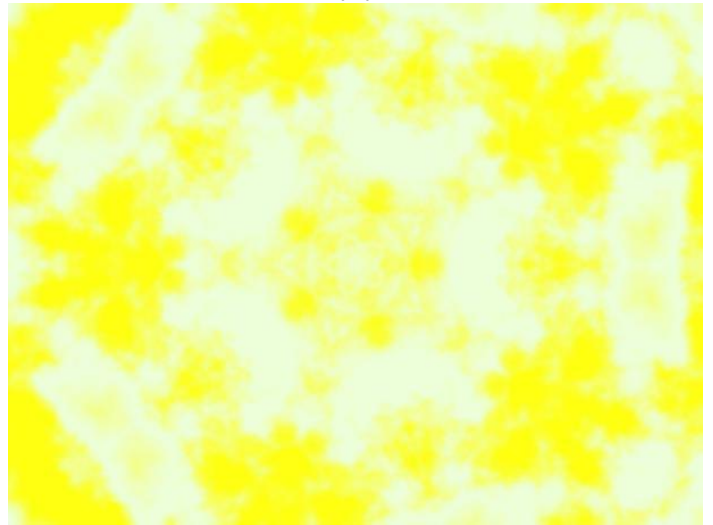

(b)

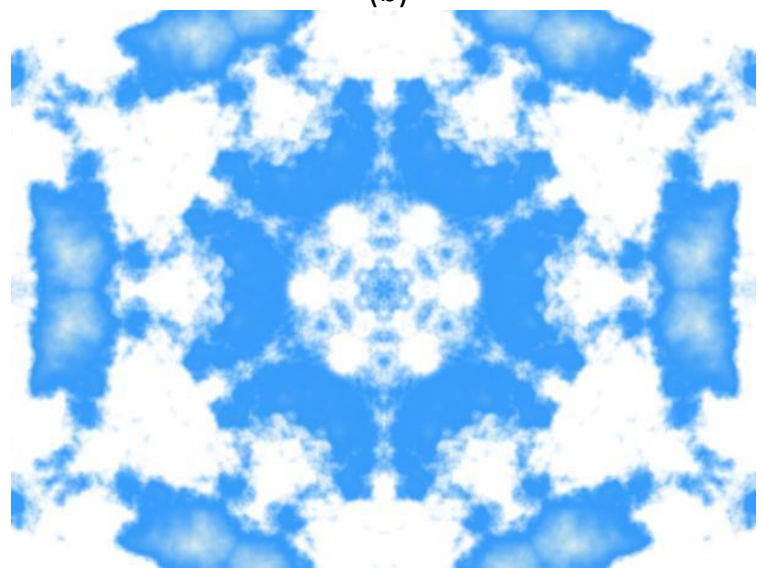

(c) 


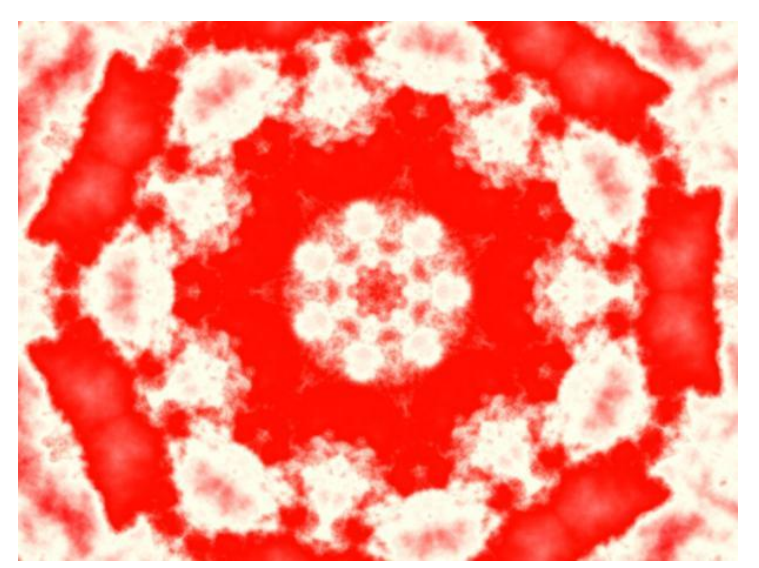

(d)

Fig3. After adjusting symmetry tie-dye patterns

Figure 3 (a) is the "Mapping" in "Symmetry Mode" is set to 4, similar to the four-leaf clover pattern; FIG. 3 (b) is the Symmetry Mode "is set to 5, similar to the five-pointed star shape; Figure 3 (b) is the Symmetry Mode "is set to 6, with snowflakes petals they seem; Fig. 3 (b) is the Symmetry Mode" is set to 7, similar to the Chinese paper-cut window grilles.

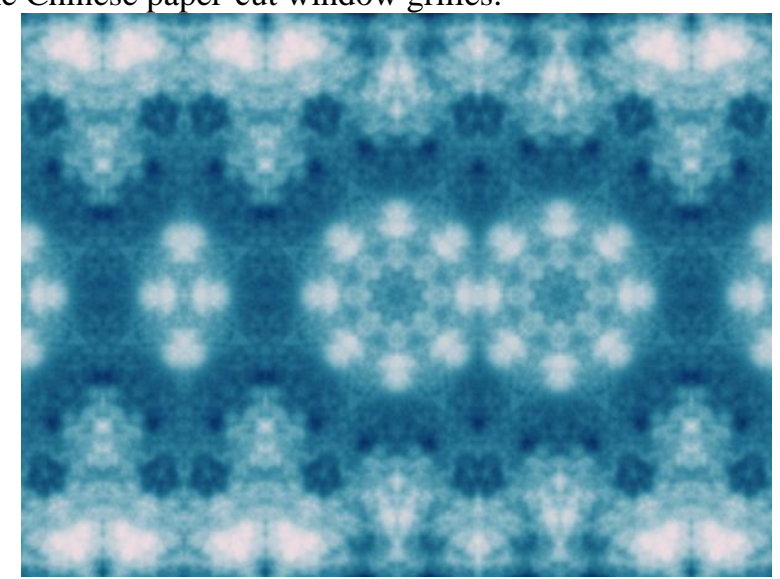

(a) 


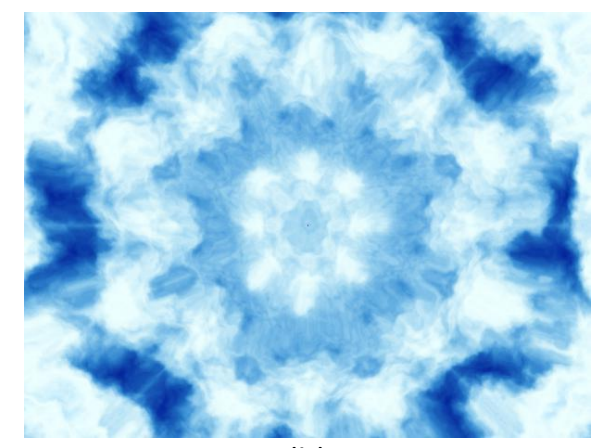

(b)

Fig.4. Add other transforms imitation tie-dye pattern

Fig. 4.(a) in the "Mapping" increased the transformation of Hexagonal Tiling 2, simulated patterns of something simple and elegant feel, and there is little traditional indigo-blue tie-dyed pattern effect. Fig. 4.(b) is in the "Mapping" added fBm Glass1 transform to simulate patterns of irregular, because the tie-dye process due to tightness in the ligation process arising from the different irregular.

\section{Summary}

Using the Kaleidoscope function of Ultra Fractal can generate tie-dye-like patterns which have symmetry regularity of designs. The kaleidoscope formula, formula and pixel clouds formulas can simulate a good tie-dye effect. Adjusting the control point gradient editor can change the color, increased color transition, to achieve the desired tie-dye effect.

\section{References}

[1] Q.P.Zhang, Tie dye patterns and its application in modern dress, J.Art experts.2007,06:206-207+49.

[2] K.J. Chen, S.B. Yang, Application of digital tie-dye patterns in fashion design, J. Popular literature.2015,08:123.

[3] S.Q. Liu, Application of Modem Tie-dye in pattern Design Based on the Computer Technology, D. Jiangnan University. 2005.

[4] S.S. Li, Computer Technology Imitate Traditional Dyeing Patterns,D. Beijing Institute of Fashion Technology.2015.

[5] J.Q. Sun, Study on fractal geometry and fractal interpolation, J. Journal of Hebei University of Technology. 2002,01:56-60.

[6] S.Y. Wang, H.F. Wang, Computer aided design of tie dye patterns, J. Textile Science and technology progress. 2009,04:48-49+56. 